\title{
Non-linear Distortion against Hearing Loss
}

\author{
Viktor BAGDÁN, Kálmán MÁTHÉ, László CZIMERMAN, József PYTEL
}

\begin{abstract}
Today, an increasing problem is hearing loss caused by increasingly high Sound Pressure Levels (SPL). Extremely loud noises and sounds originating from our environment often cause damage to our ears and result in noise-induced hearing loss (NIHL). There are procedures to increase loudness without increasing the physical, measurable Sound Pressure Level (SPL). Procedures using equal loudness sensitivity curves amplify at different frequencies. It was our intention to create a device that will control volume and clarity to a suitable level without inflicting any damage to the ear since the sound pressure level remains at a lower level. Additionally, our aim was to design a device, that, in contrast with current procedures today, does not weaken or masquerade the sound, does not distort the intricacies of music or alter tonality. Environmental conscious thinking, especially in energy saving, has become increasingly important today. When amplifying sound, we can achieve significant energy savings from utilising a lower Sound Pressure Level (SPL). Our patented method imitates the human ear's distortion and non-linear behaviour. Any non-linear distorting causes a sense of loudness growth, but the modified sound will become realistic if we come closer to the human ear's distortion. In our investigations, we conclude that, by setting (or "imitating") an overtone range similar to the human ear's distortion, we can achieve an increase in loudness in the entire audible frequency spectrum without loss of sound quality. Our prototype was constructed with the ability to carry out a subjective comparative loudness test. The data was collected individually by questionnaire, and later calculated using the arithmetical mean of the answers, with the results given in decibels. An average increment of $2.7 \mathrm{~dB}$ was measured electronically based on the 66 completed questionnaires used with the Triode Proof of Concept Model. The results demonstrated that an increase of loudness level can be achieved not only with the currently used Fletcher-Munson curves, but with the help of the procedure found in our study. In the implementation of this unique method, an increase in the sound pressure level can be avoided, and, so, without SPL increases, the subjective loudness of the sound can be increased, ensuring the ear will not be exposed to the adverse consequences of the higher sound pressure level. Our unique equipment and methodology can help reduce hearing damage without the discomfort caused by coloured tones. While further studies and improvements are required, the usability of this method demonstrates its practical applicability. These may include various electronic sound amplifiers in which the method can be used as a sound effect module. However, the most important field now using this method is huge number of media players, as young people commonly use these audio tools. The greatest benefit of this unique approach is therefore a reduction of hearing damage caused by the widespread and reckless use of headphones.
\end{abstract}

Keywords: Loudness Increase; Noise-Induced Hearing Loss (NIHL); Non-Linear Distortion; Sound Pressure Level (SPL)

\section{INTRODUCTION}

Today, an increasing problem is hearing loss caused by increasingly high Sound Pressure Levels (SPL). Extremely loud noises and sounds originating from our environment can damage our ears resulting in noise-induced hearing loss (NIHL). The receptors in the inner ear are very sensitive. Unfortunately, hair cells (hearing cells) in humans cannot be replaced if they become damaged. Hearing loss may be temporary or permanent, and, it can affect either one or both ears. In such cases of hearing loss, the clarity of speech in a noisy environment or on the telephone deteriorates. NIHL is either immediately noticeable or may become evident only later. By definition, the absolute threshold of hearing (ATH) is the minimum sound level of a pure tone that can be heard by the average human ear with normal hearing with no other sound present. The absolute threshold relates to the sound that can be first distinguished and heard by the organism [1]. A threshold shift can be temporary or permanent. A rise in the hearing threshold can be statistically justified among young people [2]. The reason for this generally relates to a noisy background, other than our natural environment. One example is the popularity of media player use, mostly used by the younger generation, often through earphones, and in particular, the auditory meatus models [3]. These media players are based on extremely efficient, class-D amplifiers, which can produce high sound pressure levels, for a long time. The equivalent sound pressure level calculated for 8 hours, which leads to permanent hearing loss, is 85 decibels, measured by an A-filter $[4,5]$. These headphones are able to reach this value, and can easily go above this level [6]. Potential danger may also occur in events, such as concerts. The human ear can tolerate values higher than $85 \mathrm{~dB}$ (A) for a short time, but not extended over 8 hours.
Another problem is the increased noise levels and noise pollution in our increasingly noisy and crowded environment. Increased noise levels are not just a problem in themselves. In fact, a certain signal to noise ratio (SNR) is necessary to make the speech understandable or to enjoy music [7]. Therefore, if the noise level increases, the signal level must be raised, which can also lead to noise-induced hearing loss.

Today, environmental-conscious thinking has become increasingly important. Public Address Systems (PASystems) used in concerts and events consume enormous amounts of power (in the tens of kilowatts range), however, the power consumed by home amplifiers is also significant due to their popularity. When amplifying sound, we can count on significant energy savings if we are satisfied with a lower Sound Pressure Level (SPL). The power change is logarithmic, so if we need a $3 \mathrm{~dB}$ increase, we need to double the power. For portable devices, power savings will result in longer battery life.

It was our intention to create a device that will control volume and clarity, yet render loudness tolerable, without inflicting any damage to the ear, since the sound pressure level remains at a lower level. Additionally, such a device should not weaken or masquerade the sound, nor distort the intricacies of music or alter tonality, unlike current procedures in use today. There are procedures that aim to increase loudness without altering the physical, measurable Sound Pressure Level (SPL). These procedures use equal loudness sensitivity curves (Fig. 1) to amplify different frequencies $[8,9]$.

A good example of this is how the perceived volume of TV commercials suddenly grew louder even though there was no change in the measurable sound pressure level that is defined by law. This phenomenon was out of the bounds of our research and instead we concentrated on 
avoiding the creation of unpleasant sound effects due to different amplifications of different frequencies.

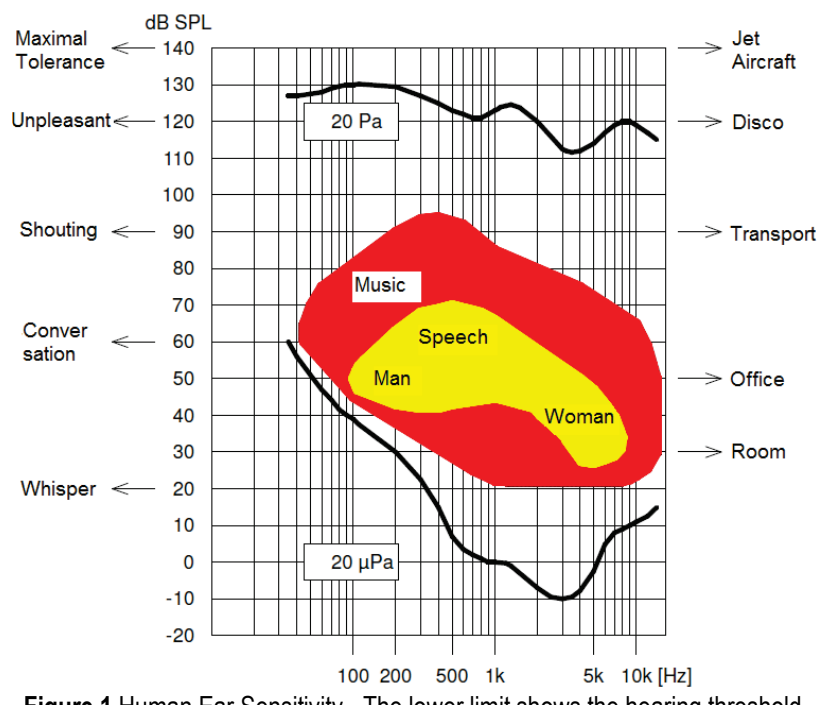

Figure 1 Human Ear Sensitivity - The lower limit shows the hearing threshold the upper limit shows the inconvenience threshold at different frequencies [9]

We believe our unique equipment and approach can help reduce hearing damage, without the discomfort caused by coloured tones.

\section{RELATED WORK}

In most cases, perceived volume increasing procedures are used to intermeddle in the original music. Okabe and Nakatoh examined the volume control system in an application to prevent the onset of headphone induced hearing loss when listening to music on a portable music player [10]. They examined the melody structure of music by decreasing the volume separately for the melody structure (Intro part, Verse, Chorus, Interlude).

Another solution can be non-linear amplification, which is widely used in hearing aids. Dynamic range compression can be helpful when the intent is to increase the volume in certain frequency ranges. As Zou, Hao and Panahi show, multi-channel compression introduces distortion to the system, and increases computational complexity. To eliminate this, they investigated a compensation filter to reduce distortion [11].

We did not find published research in the auditory field where perceived volume increases were achieved only through the correct modification of harmonics.

\section{A DEVICE FOR MODELLING HUMAN EAR DISTORTION AND A METHOD FOR SOUND SIGNAL PROCESSING 3.1 Our Sound Signal Processing Method}

This process and device is protected by patent [12]. The following diagram demonstrates the operational block diagram of the process (Fig. 2).

The patented method can precisely imitate the human ear's distortion and non-linear behaviour. Any non-linear distortion causes a sense of loudness intensity, but the modified sound will only become realistic if we come closer to the human ear's distortion. In the medium frequency range of speech and vocal voice, the ear is the most sensitive and has the lowest distortion. This range is modelled with the Linear Distortion Module "B" and then the "C" Non-linear Distortion Module enriches the sound with matching paired and odd harmonics. Finally, the "D" Inverse Linear Distortion Module restores the original tone. Amplifiers "A" and "E" can be used to adjust the optimum signal level.

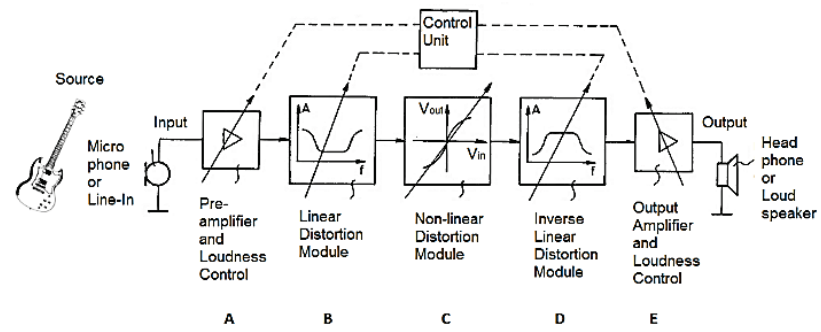

Figure 2 Block Diagram of the Sound Signal Processing Method [12]

\subsection{Prototype (PoC) for Modelling Human Ear Distortion}

A prototype was subsequently built based on the patented method. The equipment used for our research is an electronic device which does not modify the spectrum of the incoming audio frequency between $20 \mathrm{~Hz}$ and $50 \mathrm{kHz}$, instead, it modifies only the harmonics. The innovation lies in the area of quality sound amplification. In particular, the current invention is a signal processing electronic device which, while providing lower sound pressure levels, also achieves a perfectly true-to-life sound experience. In our investigations, we concluded that, by setting (or "imitating") an overtone range similar to the human ear's distortion, we can achieve a loudness increase over the entire audible frequency spectrum without loss of sound quality. Accordingly, if an electronic device for processing an audio signal would be able to create a harmonic overtone range artificially, this would avoid the use of unnecessarily high and harmful sound pressure levels in amplifying systems.

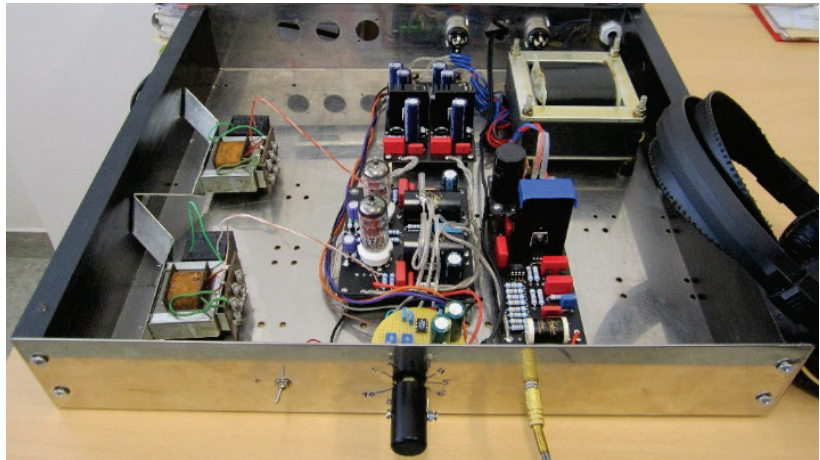

Figure 3 Prototype for Modelling the Human Ear Distortion [Máthé, Bagdán]

The prototype (Fig. 3) was constructed with the ability to undergo a subjective comparative loudness test. The device is based on a simple triode circuit, featured in Fig. 4. This allows us to switch between listening to an overtone modified sound or the unchanged sound. On the modified branch, the input and output amplitudes are equal, but the unmodified branch can be changed by a potentiometer. The clean channel is built with low distortion operation amplifiers. To make potentiometer adjustments and switch between the signal lines, it is possible to set up a similar level which corresponds with subjective listening through headphones. Once this test has been implemented, it is 
possible to infer, from the position of the potentiometer, any increase in the sense of loudness, and this can be expressed in decibels.

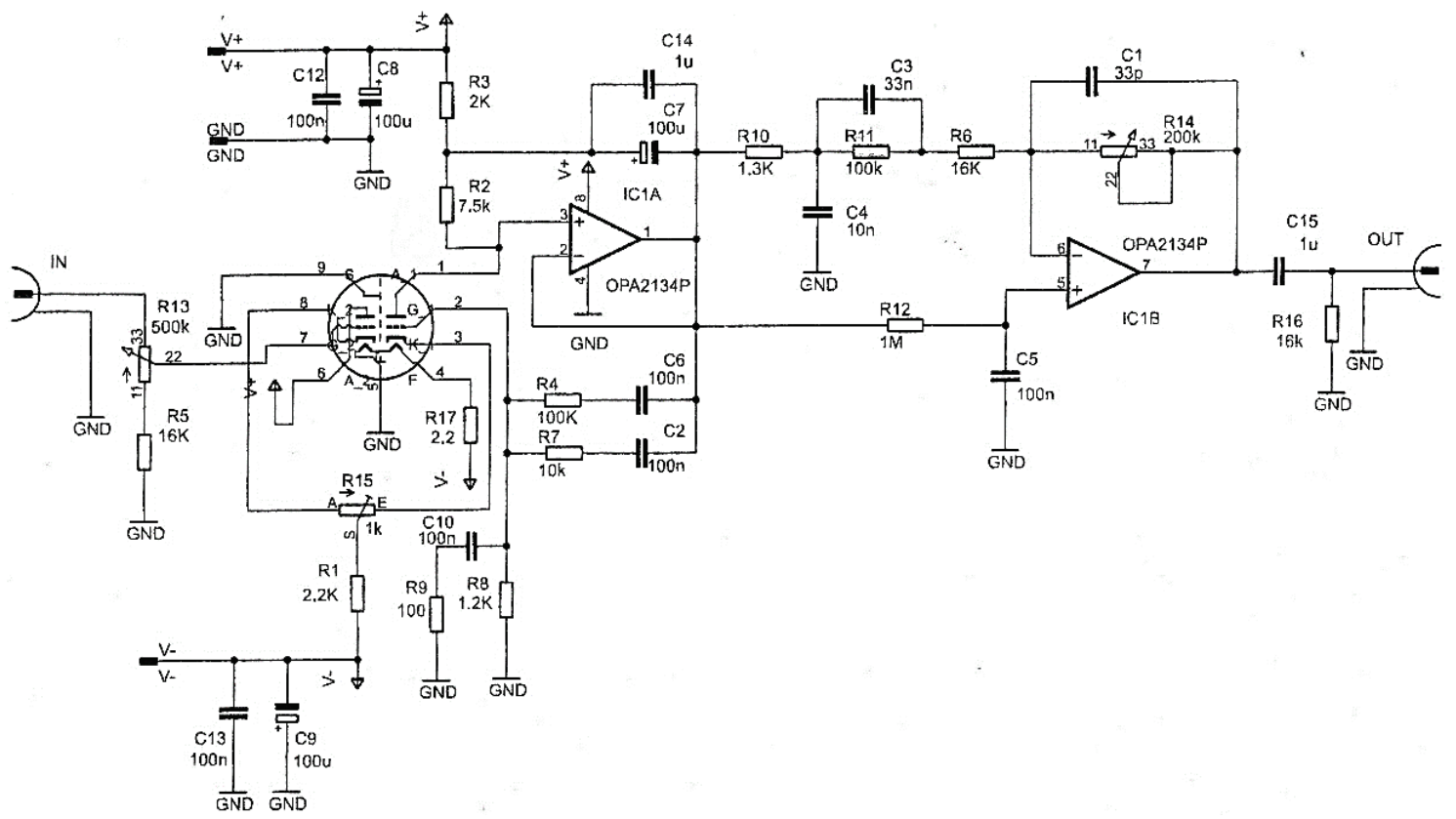

Figure 4 Triode Circuit for Modelling Human Ear Distortion [12]

\section{TESTING OF THE CONCEPT \\ 4.1 Subsequent Comparative Testing}

For the purposes of comparative testing, we used a high-resolution, compression-free and lossless, lowdistorted music sample. To ensure consistency we used a classic jazz track (Kenny Drew: Undercurrent) in SACD (Super Audio CD) format cut to two minutes. SACD is a high definition audio format with a sampling frequency of 2.8224 MHz, a 1-bit audio codec and an optical drive (disk) capacity of $4.7 \mathrm{~GB}$. The study was conducted in a mobile silent chamber in which the test leader and a volunteer were seated. The prototype was also in the chamber. The test was carried out using headphones (Sennheiser HD 430), but due to the acoustic sound pressure levels, the headphone signal was paralleled to active loudspeakers, for sound pressure level control. Sound pressure monitoring was performed using a verified measuring instrument (SVAN979 noise and vibration meter/analyser manual instrument) to which an external microphone was connected (G.R.A.S $1 / 2$ " measuring microphone). Measurement of the sound pressure level was carried out by a third individual outside the chamber. The measuring microphone sensed the sound pressure generated by the active speakers. In measuring the equivalent sound pressure level (logarithmically averaged level), we now had the opportunity to set the same sound pressure levels for both distorted and undistorted sounds.

With this method, the potentiometer position of the clean channel could also be scaled in decibels. The decibel level increase corresponding to the audible volume increase could be measured by the increase in the volume of the clean channel. For each potentiometer position, we measured how many decibels the clean channel is amplifying, using a verified sound pressure level meter (SVAN-979).

\subsection{Questionnaires}

All relevant data was collected individually by questionnaire and calculated using the arithmetical mean of the answers, with the result given in decibels. The questionnaire contained questions about whether the participant had a diagnosed hearing loss and whether they were in a noisy environment prior to performing the test. The questions and the test were compiled taking into account objective audiometry requirements [9]. The device was tested by 66 participants and students, and 66 participants effectively completed the questionnaire. Average age: 33.1 years, standard deviation: 12.1, median: 32 years, minimum: 14 years, maximum: 68 years. $36 \%$ of participants were women, $64 \%$ were men. Participation in the study was voluntary and only the results of participants who did not have hearing loss were taken into account.

\section{ANOTHER APPROACH: HARMONIC ENRICHMENT WITH A MULTIPLICATION CIRCUIT}

Another approach demonstrates how harmonics can be produced directly. This means that we can control all of the harmonic volumes together and individually. Tests using electric tube amplifiers have shown that the second, third and fourth harmonics have a major influence on the generated sound when compared with their higher-order counterparts. The following equations feature the mathematical logic we employed to create harmonics [13]. However, it is also important to note that, together with the harmonics, we also produced unnecessary components; in the form of constants and returning basic harmonics.

$$
\begin{aligned}
& f(t)=\sin (\omega t), \\
& f^{2}(t)=\frac{1}{2}-\frac{\cos (2 \omega t)}{2},
\end{aligned}
$$


$f^{3}(t)=\frac{3 \sin (\omega t)}{4}-\frac{\sin (3 \omega t)}{4}$

$f^{4}(t)=-\frac{\cos (2 \omega t)}{2}+\frac{\cos (4 \omega t)}{8}+\frac{3}{8}$,

$f^{5}(t)=\frac{10 \sin (\omega t)}{16}-\frac{5 \sin (3 \omega t)}{16}+\frac{\sin (5 \omega t)}{16}$,

$f^{6}(t)=-\frac{15 \cos (2 \omega t)}{32}+\frac{6 \cos (4 \omega t)}{32}-\frac{\cos (6 \omega t)}{32}+\frac{10}{32}$.

The multiplication function was provided by the Analog Devices AD633 chip, while the amplification was performed by the Texas Instruments NE5534 Operational Amplifier. In the final device, we implemented correction circuits and inverters. The following block diagrams (Fig. 5, Fig. 6 and Fig. 7) illustrate the operational functionality and the mathematical transformations of the equipment.

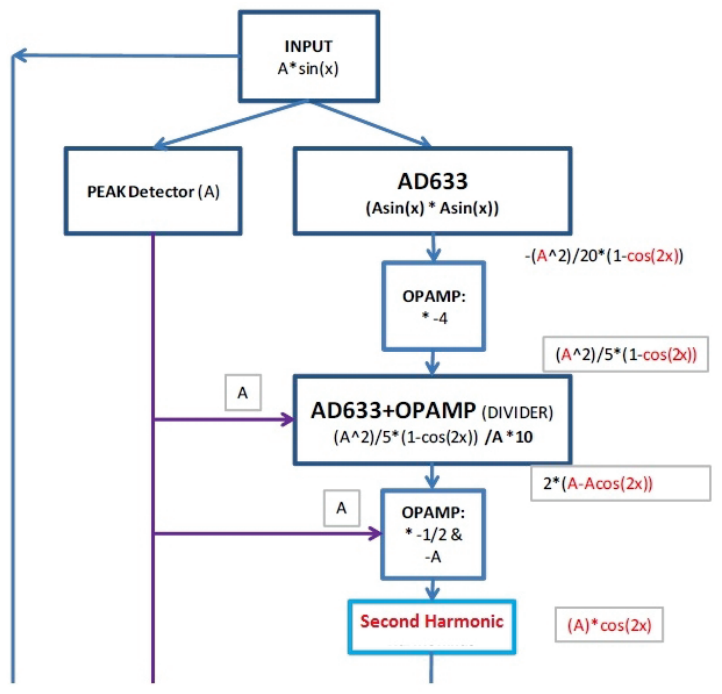

Figure 5 Block Diagram of the 2nd Harmonic Creation [13]

A circuit board diagram of the complete equipment can be seen in Fig. 8. The circuit amplitude testing was successfully performed at different frequencies.

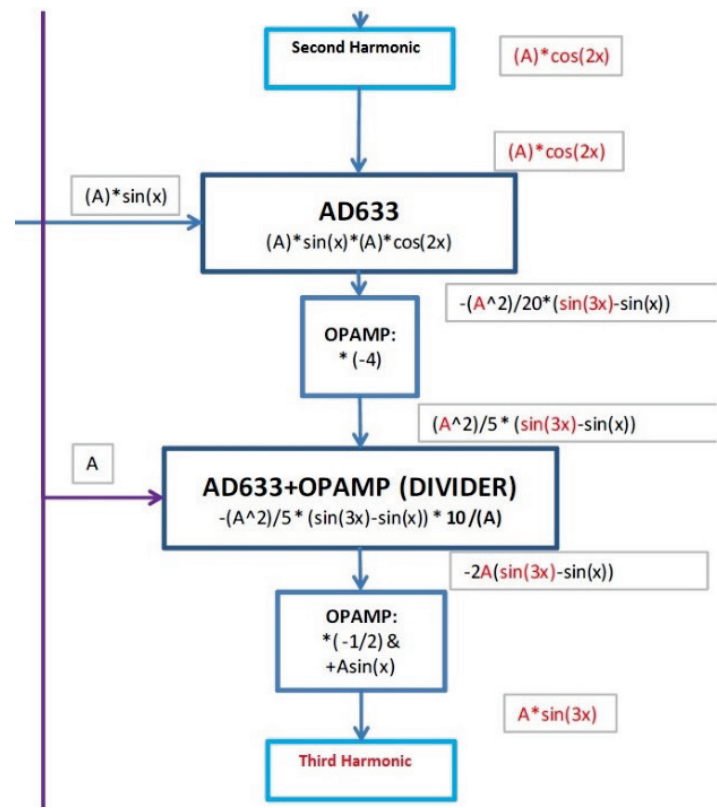

Figure 6 Block Diagram of the 3rd Harmonic Creation [13]

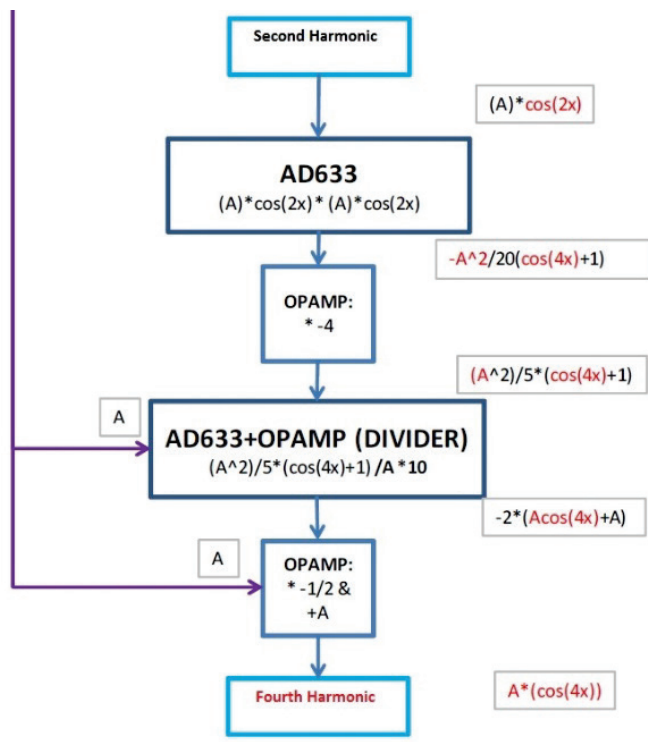

Figure 7 Block Diagram of the 4th Harmonic Creation [13]

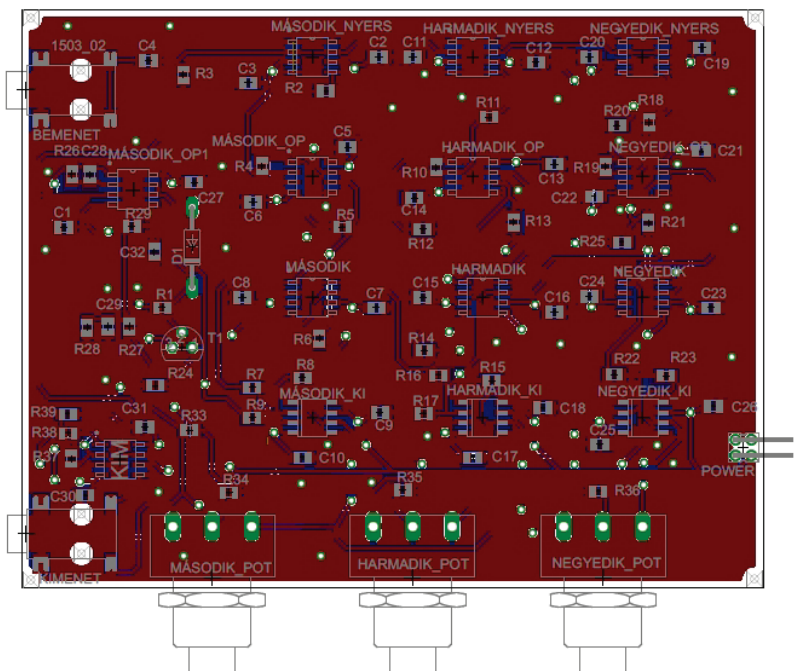

Figure 8 Diagram of the Harmonic Enrichment Circuit [13]

\section{RESULTS}

An average increment of $2.7 \mathrm{~dB}$ can be measured electronically (standard deviation: 2.2 , minimum: $-1.5 \mathrm{~dB}$, maximum $6.5 \mathrm{~dB}$, median: $2.5 \mathrm{~dB}$ ), based on the 66 completed questionnaires used with the Triode Proof of Concept Model. This represents an increase of $87.5 \%$ in terms of power change. The power increment can be calculated as follows. If we consider that input power is 1 $\mathrm{W}$, then:

$P_{0}=1 \mathrm{~W}$,

$L_{\mathrm{p}}=2.73 \mathrm{~dB}$,

$P_{1}=P_{0} \times 10^{\frac{L_{\mathrm{p}}}{10}} \mathrm{~W}=1 \mathrm{~W} \times 10^{0.273}=1.875 \mathrm{~W}$,

$\frac{P_{1}}{P_{0}}=\frac{1.875 \mathrm{~W}}{1 \mathrm{~W}}=1.875$.

This represents a power increase of $87.5 \%$. (The $3 \mathrm{~dB}$ increase is defined as a $100 \%$ power increase, which is double the power.) This means, in using a properly adjusted harmonics range, the sensed volume is higher with 
this level on average when compared to the unmodified sound, without changing the measurable sound pressure level.

Since the $2.7 \mathrm{~dB}$ increment is above the just noticeable difference (JND) of amplitude sensitivity, which is $0.5 \mathrm{~dB}$ at $80 \mathrm{~dB}$ SPL [14], our experiments demonstrate the efficiency of the process.

\section{DISCUSSION AND CONCLUSIONS}

The results clearly demonstrate that an increase in the loudness level can be achieved not only with the currently used Fletcher-Munson curves [8,9], but with the help of the procedure used in our study. In practice this means the subjective loudness of sound can be increased without any increase in the sound pressure level (SPL), meaning the ear will not be exposed to the adverse consequences of higher sound pressure level.

As Moore, Glasberg, and Stone pointed out, dynamic range compression would allow about a $58 \%$ increase in loudness for a fixed peak level [15]. This is higher than we could achieve with our prototype. Our research showed that the amplification is linear, only the manipulation of harmonics is non-linear. According to Kirchberger and Russo, the dynamic range compression, also referred to as "loudness war", has a negative effect, especially for those who use hearing aids with internal dynamics compression, so they recommend linear settings [16]. For this reason, our research is important because it is possible to achieve a similar effect without dynamic range compression.

Although additional studies and improvements are required, the versatility of this method predicts its practical applicability. These may include various electronic sound amplifiers in which the method can be used as a sound effect module. However, the most important field currently using this method is the media player, as this equipment is commonly used by the younger generation. Notably, the greatest benefit of this equipment is the reduction of hearing damage caused by the widespread and reckless use of headphones. The prototype is currently a very simple analogue circuit (a triode circuit based upon Fig. 4) so another goal is to create a digital version for adaptation to various digital devices, such as hearing aids. In further developing the prototype and by measuring the non-linear behaviour of the ear, we intend to reach a result where the modified sounds at one magnitude of lower power also produce the same volume as unmodified sounds.

\section{REFERENCES}

[1] Durrant, J. D. \& Lovrinic, J. H. (1984). Bases of hearing sciences. Second edition. United States of America: Williams \& Wilkins.

[2] Doheny, K. (2010 August 17). Hearing loss in teens is on the rise. WebMD, Retrieved from https://www.webmd.com/.

[3] Révész, P. \& Gerlinger, I. (2011). A fiatalság és az mp3 lejátszók - fokozott kockázat, kevés óvintézkedés. Otorhinolaryngologia Hungarica, 57, 182-185

[4] Widen, S. E., Båsjö, S., Möller, C., \& Kähäri, K. (2017). Headphone listening habits and hearing thresholds in swedish adolescents. Noise \& health, 19(88), 125-132. https://doi.org/10.4103/nah.NAH_65_16

[5] Lutman, M. E. (2000) What is the risk of noise-induced hearing loss at $80,85,90 \mathrm{~dB}(\mathrm{~A})$ and above? Occup. Med., 50(4), 274-275. https://doi.org/10.1093/occmed/50.4.274
[6] Fligor, B. \& Clarke Cox, L. (2005). Output levels of commercially available portable compact disc players and the potential risk to hearing. Ear and Hearing, 25(6), 51327. https://doi.org/10.1097/00003446-200412000-00001

[7] Meyer, J., Dentel, L., \& Meunier, F. (2013). Speech recognition in natural background noise. PLOS ONE, 8(11), e79279. https://doi.org/10.1371/journal.pone.0079279

[8] Fletcher, H. \& Munson, W. A. (1933). Loudness, its definition, measurement and calculation. Journal of the Acoustical Society of America, 5, 82-108. https://doi.org/10.1121/1.1915637

[9] Pytel, J. (1996). Audiológia. Pécs, Hungary: Victoria Kft.

[10] Okabe, D. \& Nakatoh, Y. (2018). Study on headphone hearing loss prevention methods based on the melody structure of music on portable music player. 2018 IEEE International Conference on Consumer Electronics (ICCE), Las Vegas, NV, 1-2. https://doi.org/10.1109/ICCE.2018.8326297

[11] Zou, Z., Hao, Y. \& Panahi, I. (2018). Design of compensated multi-channel dynamic-range compressor for hearing aid devices using polyphase implementation. $201840^{\text {th }}$ Annual International Conference of the IEEE Engineering in Medicine and Biology Society (EMBC), Honolulu, HI, 429432. https://doi.org/10.1109/EMBC.2018.8512441

[12] Bagdan, V., Czimerman, L., Mathe, K., \& Pytel, J. (2014). European Patent No. WO2014041385. Retrieved from https://worldwide.espacenet.com/publicationDetails/biblio? $\mathrm{II}=0 \& \mathrm{ND}=3 \&$ adjacent $=$ true $\&$ locale $=$ en $E P \& \mathrm{FT}=\mathrm{D} \&$ date $=$ $20140320 \& C C=$ WO $\& N R=2014041385 \mathrm{~A} 2 \& \mathrm{KC}=\mathrm{A} 2$

[13] Gardánfalvi, A. (2016). Harmonikus dúsító áramkör tervezése a fül nemlineáris viselkedésének tanulmányozásához. Degree Thesis. Retrieved from University of Pécs, Faculty of Engineering and Information Technology Database.

[14] Jesteadt, W., Weir, C. C., \& Green, D. M. (1977). Intensity discrimination as a function of frequency and sensation level. JASA, 61, 169-177. https://doi.org/10.1121/1.381278

[15] Moore, B., Glasberg, B., \& Stone, M. A. (2003). Why are commercials so loud? - Perception and modeling of the loudness of amplitude-compressed speech. AES: Journal of the Audio Engineering Society, 51, 1123-1132.

[16] Kirchberger, M. \& Russo, F. A. (2016). Dynamic range across music genres and the perception of dynamic compression in hearing-impaired listeners. Trends in Hearing. https://doi.org/10.1177/2331216516630549

\section{Contact information:}

Viktor BAGDÁN, assistant lecturer

(Corresponding author)

Doctoral School of Health Sciences, University of Pécs,

Vörösmarty Mihály utca 4, H-7621 Pécs, Hungary

bagdan.viktor@mik.pte.hu

Kálmán MÁTHÉ, retired assistant professor

Faculty of Engineering and Information Technology, University of Pécs, Boszorkány út 2, H-7624 Pécs, Hungary

mathe.kalman@mik.pte.hu

László CZIMERMAN, retired technical assistant

Faculty of Engineering and Information Technology, University of Pécs,

Boszorkány út 2, H-7624 Pécs, Hungary

czimerman.laszlo@mik.pte.hu

József PYTEL, Professor Dr.

ENT Department (Department of Otorhinolaryngology), University of Pécs, Munkácsy Mihály utca 2, H-7621 Pécs, Hungary

pytel.jozsef@pte.hu 